\title{
Superconductivity in a doped Mott insulator
}

\author{
Dung-Hai Lee \\ Department of Physics, University of California at Berkeley, Berkeley, CA 94720, USA
}

\begin{abstract}
Starting from the d-wave RVB mean-field theory of Kotliar and Liu, we present a new, longwavelength/low-energy exact, treatment of gauge fluctuations. The result is a theory of gapless fermion quasiparticles coupled to superconducting phase fluctuations. We will discuss the physical implications, and the similarity/differences with a theory of BCS pairing with phase fluctuations.
\end{abstract}

PACS numbers: 74.25.Jb, 79.60.-i, 71.27.+a

The high-temperature superconductors are doped Mott insulators. Shortly after the experimental report of high $T_{c}$, Anderson proposed the "resonating-valencebond"(RVB) idea. [1] According to this idea the root of high temperature superconductivity is an insulating spin liquid of singlet pairs. Due to Coulomb blockade these pairs are unable to move. Doping creates electronic vacancies that mobilize these pairs. Once mobile, the valence-bond pairs can Bose condense into a superconducting state.

From the beginning the Neel-ordered state found in the undoped cuprates presents a difficulty to the RVB idea. Motivated by the experimental fact that the Neel order is destroyed once the sample becomes metallic/superconductive, there is a general hope that sufficient doping stablizes the long-sort (doped) spin liquid.

The notion of RVB is made very attractive due to the experimental data on the "underdoped cuprates". For example there exists a significant temperature range $\left(T^{*}>T>T_{c}\right)$ where a pseudogap exists in the spin excitation spectrum when there is no superconductivity. Angle-resolved photoemission spectroscopy (ARPES) [2] revealed the fact that the size and the momentum dependence of the pseudogap is roughly the same as the d-wave gap in the superconducting state. In addition, ARPES studies of undoped cuprates find a peak above the Mott-Hubbard gap with a dispersion similar to that of spinons [3] in the RVB mean-field theories. [4.5]

On the theory side, implementation of RVB beyond mean-field theories [5] [9] is hindered by the necessity to treat strong gauge fluctuations. [6 8] In the absence of a reliable treatment of the gauge field, it is impossible to tell which features of the mean-field theory actually correspond to reality. In this paper we revisit the KotliarLiu d-wave RVB mean-field theory. [5] What we were able to find is an exact treatment of gauge fluctuations at long wavelength and low energy. Eqs.(12,13) plus the discussions after it 1-6) are the main results of this paper.

Our starting point is the following boson-fermion representation of the $\mathrm{t}-\mathrm{J}$ model: [8]

$$
Z=\int D[U] D\left[a_{0}\right] D\left[\psi^{+}, \psi\right] D\left[b^{+}, b\right] e^{-\int d t \mathcal{L}},
$$

where

$$
\begin{aligned}
\mathcal{L} & =\sum_{i j}\left[\frac{J}{2} \operatorname{Tr}\left(U_{i j}^{+} U_{i j}\right)-i a_{0 i} \delta_{i j}\right]+\frac{1}{2} \sum_{i j} \psi_{i \sigma}^{+}\left[\left(\partial_{0}+i a_{0 i} \tau_{z}\right) \delta_{i j}\right. \\
& \left.+J U_{i j}\right] \psi_{j \sigma}+\sum_{i j} b_{i}^{+}\left[\left(\partial_{0}+i a_{0 i}-\mu\right) \delta_{i j}-t \chi_{i j}\right] b_{j}
\end{aligned}
$$

Here $i, j$ runs through the sites of a square lattice, $\psi_{i \sigma}=$ $\left(\begin{array}{c}f_{i \sigma} \\ \epsilon_{\sigma \sigma^{\prime}} f_{i \sigma^{\prime}}^{+}\end{array}\right), \tau_{z}=\left(\begin{array}{cc}1 & 0 \\ 0 & -1\end{array}\right), U_{i j} \equiv\left(\begin{array}{cc}-\chi_{i j}^{*} & \Delta_{i j}^{*} \\ \Delta_{i j} & \chi_{i j}\end{array}\right)$, and the rest of the notations are standard. In the literature $b_{i}$ and $f_{i \sigma}$ are often referred to as holon and spinon annihilation operators respectively.

First, let us briefly review the results of mean-field theory. [5, 9] For $x>0$, the mean-field solutions are characterized by the following parameters: $i a_{0 i}(t) \rightarrow \mu_{f}$, $b_{i}(t) \rightarrow b, \chi_{i j}(t) \rightarrow \chi_{0}$, and $\Delta_{i j}(t) \rightarrow \Delta_{0} \eta_{i j}$. Here $\eta_{i j}=+1$ if $i, j$ are are $\hat{x}$-nearest-neighbor, and $=-1$ if $i, j$ are $\hat{y}$-nearest-neighbor. When the temperature is not too high, both $\mu_{f}$ and $\chi_{0}$ are nonzero. Depending on the values of $b$ and $\Delta_{0}$ the low temperature $x-T$ plane is divided into four regions: i) d-wave superconducting phase where $\Delta_{0} \neq 0$, and $b \neq 0$, ii) the spin gap phase where $\Delta_{0} \neq 0$, and $b=0$, iii) the Fermi liquid phase where $\Delta_{0}=0$, and $b \neq 0$, and iv) the strange metal phase where $\Delta_{0}=b=0$. The boundaries between these four phases are $T_{B E}(x)$ and $T_{\text {pair }}(x)$ at which $b$ and $\Delta_{0}$ develop expectation values respectively. In mean-field theory both $T_{B E}(x)$ and $T_{\text {pair }}(x)$ mark phase transitions. This statement will be made invalid by fluctuations. A schematic phase diagram can be found in, e.g., Ref. [10].

According to the phase diagram [10] $T_{\text {pair }}(x)>$ $T_{B E}(x)$ in the underdoped regime. Throughout this regime there exists a temperature range $T_{B E}(x)<T<$ $T_{\text {pair }}(x)$ in which a spin gap exists without superconductivity. In the rest of the paper, we shall concentrate on the d-wave superconducting phase and the spin gap regime mentioned above.

At temperatures much lower than $T_{\text {pair }}$ the important fluctuations include: i) the gapless spinon excitations and ii)

$$
\begin{aligned}
& \chi_{i j} \rightarrow \chi_{0} e^{i a_{i j}}, \quad \Delta_{i j} \rightarrow \Delta_{0} \eta_{i j} e^{i \theta_{s p, i j}} \\
& b_{i} \rightarrow \sqrt{\rho_{b, i}} e^{i \theta_{b, i}}, \quad a_{0 i} \rightarrow-i \mu_{f}+a_{0 i} .
\end{aligned}
$$


Among these fluctuations $a_{0 i}$ and $a_{i j}$ act to enforce the no-double-occupancy constraint. More explicitly $a_{0 i}$ enforces $f_{i \sigma}^{+} f_{i \sigma}+b_{i}^{+} b_{i}=1$, while $a_{i j}$ ensures no net flow of the boson and fermion current. In the following we present a new approach that treats the $a_{0 i}$ and $a_{i j}$ fluctuations (hence the constraint) exactly at long wavelength and low energy.

First let us write down a continuum action (its microscopic cutoff length is the holon separation) to capture all the fluctuations discussed above:

$$
\begin{aligned}
\mathcal{L} & =\psi_{b}^{+}\left[\left(\partial_{0}+i a_{0}-i A_{0}\right)+\frac{1}{2 m_{b}}|\mathbf{p}+\mathbf{a}-\mathbf{A}|^{2}\right] \psi_{b} \\
& +\frac{u_{b}}{2}\left(\psi_{b}^{+} \psi_{b}-\bar{\rho}_{b}\right)^{2}+\frac{K_{s p}}{2}\left|\phi_{s p}^{*}\left(\frac{\nabla}{i}+2 \mathbf{a}\right) \phi_{s p}\right|^{2} \\
& +\frac{1}{2 u_{s p}}\left(\phi_{s p}^{*} \frac{\partial_{0}}{i} \phi_{s p}+2 a_{0}\right)^{2}+i \bar{\rho}_{s p}\left(\phi_{s p}^{*} \frac{\partial_{0}}{i} \phi_{s p}+2 a_{0}\right) \\
& +i J_{f \mu}\left(\phi_{s p}^{*} \frac{\partial_{\mu}}{i} \phi_{s p}+2 a_{\mu}\right)-i \bar{\rho} a_{0}+\mathcal{L}_{f}\left[\Delta_{0}\right]
\end{aligned}
$$

In the above $\psi_{b}=\sqrt{\rho_{b}} e^{i \theta_{b}} \equiv \sqrt{\rho_{b}} \phi_{b}$ is the holon field, $\phi_{s p}=e^{i \theta_{s p}}$ is the phase factor associated with the spinon pairs, $\bar{\rho}_{b}$ and $\bar{\rho}_{s p}$ are the average densities of holon and spinon pairs, $\bar{\rho}$ is the density at half-filling, $m_{b}$ is the holon effective mass, $A_{\mu}$ is the physical gauge field, $\mathcal{L}_{f}$ is the Dirac quasiparticle action which depends on the pairing amplitude $\Delta_{0}$, $J_{f}=\left(\frac{1}{2} \sum_{n} \Psi_{n \sigma}^{+} \tau_{z} \Psi_{n \sigma}, \frac{i}{2} v_{F} \Psi_{1 \sigma}^{+} \Psi_{1 \sigma}, \frac{i}{2} v_{F} \Psi_{2 \sigma}^{+} \Psi_{2 \sigma}\right)$ is the 3 -current of the spinon quasiparticles, where $\Psi_{n \sigma}(n=$ 1,2) are the spinon Nambu spinors associated with the two Dirac points respectively. We note that the spinon and spinon-pair parts of Eq. (何) are the action of a phase fluctuating BCS superconductor. [1]

By substituting $\psi_{b}=\sqrt{\rho_{b}} \phi_{b}$ into Eq. (1) we obtain

$$
\begin{aligned}
\mathcal{L} & =\frac{\rho_{b}}{2 m_{b}}\left|\phi_{b}^{*}\left(\frac{\nabla}{i}+\mathbf{a}-\mathbf{A}\right) \phi_{b}\right|^{2}+\frac{\left|\nabla \rho_{b}\right|^{2}}{8 m_{b} \rho_{b}}+\frac{u_{b}}{2}\left(\rho_{b}-\bar{\rho}_{b}\right)^{2} \\
& +\frac{K_{s p}}{2}\left|\phi_{s p}^{*}\left(\frac{\nabla}{i}+2 \mathbf{a}\right) \phi_{s p}\right|^{2}+\frac{1}{2 u_{s p}}\left(\phi_{s p}^{*} \frac{\partial_{0}}{i} \phi_{s p}+2 a_{0}\right)^{2} \\
& +i \delta \rho_{b}\left(\phi_{b}^{*} \frac{\partial_{0}}{i} \phi_{b}+a_{0}-A_{0}\right)+i J_{f \mu}\left(\phi_{s p}^{*} \frac{\partial_{\mu}}{i} \phi_{s p}+2 a_{\mu}\right) \\
& -2 i \bar{\rho}_{s p}\left(\phi_{b}^{*} \frac{\partial_{0}}{i} \phi_{b}-A_{0}\right)+i \bar{\rho}_{s p} \phi_{s p}^{*} \frac{\partial_{0}}{i} \phi_{s p} \\
& -i \bar{\rho} A_{0}+\mathcal{L}_{f}\left[\Delta_{0}\right],
\end{aligned}
$$

where $\delta \rho_{b} \equiv \rho_{b}-\bar{\rho}_{b}$. In obtaining the above we have used the fact that due to the lattice effect (i.e. when viewed by the vortices, density $\bar{\rho}$ of bosons can be gauged away because it corresponds to one flux quantum per plaquette) $i \bar{\rho}_{b} \phi_{b}^{*} \frac{\partial_{0}}{i} \phi_{b}=-2 i \bar{\rho}_{s p} \phi_{b}^{*} \frac{\partial_{0}}{i} \phi_{b}$.

Next we Hubbard-Stratonavich decouple the first, the fourth, and the fifth terms to obtain

$$
\begin{aligned}
\mathcal{L} & =\frac{m_{b}}{2 \rho_{b}}\left|\mathbf{j}_{b}\right|^{2}+\frac{u_{b}}{2} \delta \rho_{b}^{2}+\frac{\left|\nabla \rho_{b}\right|^{2}}{8 m_{b} \rho_{b}}+\frac{1}{2 K_{s p}}\left|\mathbf{j}_{s p}\right|^{2}+\frac{u_{s p}}{2} \delta \rho_{s p}^{2} \\
& +i\left(J_{s p \mu}+J_{f \mu}\right)\left(\phi_{s p}^{*} \frac{\partial_{\mu}}{i} \phi_{s p}+2 a_{\mu}\right)
\end{aligned}
$$

$$
\begin{aligned}
& +i J_{b \mu}\left(\phi_{b}^{*} \frac{\partial_{\mu}}{i} \phi_{b}+a_{\mu}-A_{\mu}\right)-2 i \bar{\rho}_{s p}\left(\phi_{b}^{*} \frac{\partial_{0}}{i} \phi_{b}-A_{0}\right) \\
& +i \bar{\rho}_{s p} \phi_{s p}^{*} \frac{\partial_{0}}{i} \phi_{s p}-i \bar{\rho} A_{0}+\mathcal{L}_{f}\left[\Delta_{0}\right] .
\end{aligned}
$$

In Eq. (6) $J_{b \mu} \equiv\left(\delta \rho_{b}, \mathbf{j}_{b}\right), J_{s p \mu} \equiv\left(\delta \rho_{s p}, \mathbf{j}_{s p}\right)$ where $\mathbf{j}_{b}$, $\mathbf{j}_{s p}$ and $\delta \rho_{s p}$ are the auxiliary field introduced by the Hubbard-Stratonavich transformation. Physically $J_{b \mu}$ and $J_{s p \mu}$ are the three-currents of the holon and spinon pairs. In the following, for distances greater than the holon separation $(\sim 1 / \sqrt{x})$, we shall linearize Eq. (6) by replacing $\rho_{b}$ in the first term by $\bar{\rho}_{b}$ and drop the third term. The resulting action read

$$
\begin{aligned}
\mathcal{L} & =\frac{1}{2 K_{b}}\left|\mathbf{j}_{b}\right|^{2}+\frac{u_{b}}{2} \delta \rho_{b}^{2}+\frac{1}{2 K_{s p}}\left|\mathbf{j}_{s p}\right|^{2}+\frac{u_{s p}}{2} \delta \rho_{s p}^{2} \\
& +i\left(J_{s p \mu}+J_{f \mu}\right)\left(\phi_{s p}^{*} \frac{\partial_{\mu}}{i} \phi_{s p}+2 a_{\mu}\right) \\
& +i J_{b \mu}\left(\phi_{b}^{*} \frac{\partial_{\mu}}{i} \phi_{b}+a_{\mu}-A_{\mu}\right)+\mathcal{L}_{f}\left[\Delta_{0}\right] \\
& -2 i \bar{\rho}_{s p}\left(\phi_{b}^{*} \frac{\partial_{0}}{i} \phi_{b}-A_{0}\right)+i \bar{\rho}_{s p} \phi_{s p}^{*} \frac{\partial_{0}}{i} \phi_{s p}-i \bar{\rho} A_{0} .
\end{aligned}
$$

In the above $K_{b} \equiv \frac{\bar{\rho}_{b}}{m_{b}}$ and $K_{s p} \equiv \frac{\bar{\rho}_{s p}}{m_{s p}}$.

Now we can integrate out $a_{\mu}$ exactly. The result is the constraint

$$
J_{b \mu}+2\left(J_{s p \mu}+J_{f \mu}\right)=0,
$$

i.e. the total three current with respect to $a_{\mu}$ is zero! Physically this is due to the no-double-occupancy constraint which requires each lattice site to be occupied by either a holon or a spinon. Since they both carry the same charge with respect to $a_{\mu}$, the total charge is constant at any time. As the result the total three-current vanishes. If we define $J_{p \mu} \equiv\left(\delta \rho_{p}, \mathbf{j}_{p}\right) \equiv \frac{1}{2} J_{b \mu}$, Eq. (8) implies

$$
J_{s p \mu}=-J_{p \mu}-J_{f \mu} .
$$

Substitute Eq. (9) into Eq. (6) we obtain

$$
\begin{aligned}
\mathcal{L} & =\frac{1}{2}\left(\frac{4}{K_{b}}+\frac{1}{K_{s p}}\right)\left|\mathbf{j}_{p}\right|^{2}+\frac{1}{2}\left(4 u_{b}+u_{s p}\right) \delta \rho_{p}^{2} \\
& +i J_{p \mu}\left(\phi_{p}^{*} \frac{\partial_{\mu}}{i} \phi_{p}-2 A_{\mu}\right)+\frac{1}{K_{s p}} \mathbf{j}_{p} \cdot \mathbf{j}_{f}+u_{s p} \delta \rho_{p} \rho_{f} \\
& +\frac{1}{2 K_{s p}}\left|\mathbf{j}_{f}\right|^{2}+\frac{u_{s p}}{2} \rho_{f}^{2}-i \bar{\rho}_{s p}\left(\phi_{p}^{*} \frac{\partial_{0}}{i} \phi_{p}-2 A_{0}\right) \\
& +\mathcal{L}_{f}\left[\Delta_{0}\right]-i \bar{\rho} A_{0} .
\end{aligned}
$$

In the above

$$
\phi_{p} \equiv \phi_{s p}^{*} \phi_{b}^{2}
$$

Now we can integrate out $J_{p \mu}$ to obtain 


$$
\begin{aligned}
\mathcal{L} & =\frac{K_{p}}{2}\left|\phi_{p}^{*} \frac{\nabla}{i} \phi_{p}-2 \mathbf{A}\right|^{2}+\frac{1}{2 u_{p}}\left(\phi_{p}^{*} \frac{\partial_{0}}{i} \phi_{p}-2 A_{0}\right)^{2} \\
& -i z_{j} \mathbf{j}_{f} \cdot\left(\phi_{p}^{*} \frac{\nabla}{i} \phi_{p}-2 \mathbf{A}\right)-i z_{\rho} \rho_{f}\left(\phi_{p}^{*} \frac{\partial_{0}}{i} \phi_{p}-2 A_{0}\right) \\
& -i \bar{\rho}_{s p}\left(\phi_{p}^{*} \frac{\partial_{0}}{i} \phi_{p}-2 A_{0}\right)+\mathcal{L}_{f}^{\prime}\left[\Delta_{0}\right] .
\end{aligned}
$$

In the above

$$
\begin{aligned}
& K_{p} \equiv \frac{1}{K_{s p}^{-1}+4 K_{b}^{-1}}, \quad u_{p} \equiv u_{s p}+4 u_{b} \\
& z_{j} \equiv \frac{K_{s p}^{-1}}{K_{s p}^{-1}+4 K_{b}^{-1}}, \quad z_{\rho} \equiv \frac{u_{s p}}{u_{s p}+4 u_{b}},
\end{aligned}
$$

and

$$
\mathcal{L}_{f}^{\prime}\left[\Delta_{0}\right] \equiv \mathcal{L}_{f}\left[\Delta_{0}\right]+\frac{2}{K_{s p}+4 K_{b}}\left|\mathbf{j}_{f}\right|^{2}+\frac{2}{u_{s p}^{-1}+4 u_{b}^{-1}} \rho_{f}^{2},
$$

Eqs (12 14) is the main result of this paper. Aside from the renormalization factor $z_{\rho}$ and $z_{j}$ and the quasiparticle interaction in Eq. (14) the form of Eq. (12) agrees with that written down in Ref. [11] based on weak-coupling considerations. In the following we comment on several salient features/consequences of Eqs.(12)14).

1.Quasiparticle interaction: The last two terms in Eq. (14) correspond to quasiparticle interaction. Since both of them are local and have scaling dimension 4 with respect to the free Dirac theory, they are irrelevant in the renormalization group sense. In addition both coupling constants $\frac{2}{K_{s p}+4 K_{b}}$ and $\frac{2}{u_{s p}^{-1}+4 u_{b}^{-1}}$ stays finite as $x \rightarrow 0$. (As $x \rightarrow 0$ we expect $K_{b} \sim x$, while $K_{s p}, u_{b}$ and $u_{s p}$ all stay finite.) For these reasons we believe that one can safely neglect the quasiparticle interaction.

In the superconducting phase, one can further integrate out the gaussian phase fluctuations in $\phi_{s p}$. The generated spinon quasiparticle interaction is again local, and irrelevant. This result suggests that in the superconducting state the spin degrees of freedom are asymptotically described by a free Dirac theory.

2.Quasiparticle charge and current renormalization: An important effect of the no-double-occupancy constraint is to introduce renormalization factors $z_{\rho}$ and $z_{j}$. We emphasize that when $z_{j} \neq 1$ and $z_{\rho} \neq 1$, Eq. (12) is not equivalent to a BCS superconductor with phase fluctuations. 11, 12] Given the $x$-dependence of $K_{b, s p}$ and $u_{b, s p}$, it is easy to show that $z_{j} \sim x$ while $z_{\rho} \sim 1$. With such $x$-dependent $z_{j}$ Eq. (12) does not have the desired form written down in Ref. 13] to explain the temperature derivative of superfluid density. Indeed, according to the present result the suppression of the superfluid density due to thermal quasiparticle current fluctuation will be proportional to $x^{2} T$. 14, 10] It is interesting to note that since $z_{\rho} \sim 1$ we expect an x-independent linear temperature correction to $u_{p}$ due to quasiparticle screening.
It is worth emphasizing that in deriving Eq. (12) we have not assumed holon condensation, and that Eqs.(12,13,14) are applicable in both $\phi_{p^{-}}$-coherent and $\phi_{p^{-}}$ incoherent phases.

3.Flux quantization: After integrating out $a_{\mu}$, only the combination $\phi_{p}=\phi_{b}^{2} \phi_{s p}^{*}$ of the holon and the spinonpair phase factors appear in Eq. (12). We emphasize that the appearance of $\phi_{b}^{2}$ in the above expression is not due to holon pairing. This is important because in the presence of Coulomb interaction, and in the absence of screening at distances smaller than inter-holon separation, such pairing will be highly energetically unfavorable. The reason that $\phi_{b}^{2}$ appears in $\phi_{p}$ is the fact that the movement of a spinon pair always causes two holons to relocate. The combination $\left(\phi_{p}^{*} \frac{\partial_{\mu}}{i} \phi_{p}-2 A_{\mu}\right)$ in Eq. (12) (note the coefficient 2 in front of $A_{\mu}$ ) implies that in the superconducting state (i.e. $\phi_{p}$ orders) magnetic flux will be quantized in units of $h c / 2 e$.

4.The pseudogap phase: Under the present framework the superconducting state corresponds to the $\phi_{p^{-}}$ ordered phase, but how to view the pseudogap regime? According to the RVB mean-field theory, such phase arises from holon uncondensing. This remains qualitatively true in the present results. For small $x$ where the superfluid density is low, there is a wide temperature range $T_{c}<T<T_{\text {pair }}$ in which $\phi_{p}$ is thermally disordered while the spinons remain paired. In this temperature range there is no superconductivity but shows a d-wave gap for spin excitations. Clearly, it is tempting to associate this crossover regime with the pseudogap regime seen experimentally.

Due to the wide separation between $T_{c}(\sim x)$ and $T_{\text {pair }}$, there could be intermediate temperatures at which the thermal correlation length of $\phi_{p}$ is comparable with the microscopic cutoff $\sim 1 / \sqrt{x}$ while is still much larger than the spinon pairing length $\sim v_{F} / \Delta_{0}$. At these temperatures the "bare" superfluid density will vanish without destroying the spin pseudogap. [15]

In the literature there exists a debate as to whether the pseudogap regime can be described as a phase-disordered superconductor. 20 22, 10] Eq. (12) gives an affirmative answer to this question. However, as pointed out earlier, due to $z_{j}, z_{\rho} \neq 1$ and the discussion below, Eq. (12) does not describe an ordinary BCS superconductor with phase fluctuations.

Lastly, since we have integrated out the gauge field exactly, the issue of whether the gauge fluctuations destabilize the pseudogap regime [16] does not arise. [17]

5.Spin-charge separation: Eq. (12) exhibits spincharge separation in the sense that the low-energy spinon excitations are largely determined by the RVB mean-field theory, and are little affected by the gauge fluctuations. On the other hand the charge response depends on quantity such as $K_{p}$ which is greatly affected by the gauge fluctuations. A concrete example of this separation is that the vortex core size will be very different from spinon 
pairing length scale $\left(\sim v_{F} / \Delta_{0}\right)$.

To calculate the size of vortex core we restore the $\frac{\left|\nabla \rho_{b}\right|^{2}}{8 m_{b} \rho_{b}}$ term in Eq. (6). Repeating the calculations between Eq. (7) to Eq. (12) we obtain the following (most relevant) addition to Eq. (12):

$$
\Delta \mathcal{L}=\frac{1}{2 m_{b} \bar{\rho}_{b}}\left|\nabla \rho_{p}\right|^{2},
$$

Using this result we obtain a vortex core size given by

$$
\frac{1}{\xi^{2}}=\frac{u_{s p}+4 u_{b}}{\frac{1}{m_{b} \bar{\rho}_{b}}} \sim x .
$$

Thus as $x \rightarrow 0$ the vortex core size diverge as $\frac{1}{\sqrt{x}}$. In sharp contrast, the size of spinon pair $\left(v_{F} / \Delta_{0}\right)$ does not depend on $x$. A further manifestation of the spin-charge separation is in the structure of vortex core described in the following.

6. Vortex core: Near a vortex core $\bar{\rho}_{b}$ become spatial dependent so that $\bar{\rho}_{b}(\mathbf{x}) \rightarrow 0$ toward the center of the vortex. Under such condition $K_{b}$ in Eq. (13) becomes spatial dependent and $K_{b}(\mathbf{x})=\frac{\bar{\rho}_{b}(\mathbf{x})}{m_{b}} \rightarrow 0$ toward the center of the vortex. As the result both $K_{p}$ and $z_{j}$ vanishes in the vortex core. On the contrary the spinon excitations (which depend on the mean-field pairing amplitude $\Delta_{0}$ ) remain largely unaffected by the depletion of superfluid density. 18 (To be more precise, since holons are depleted from the vortex core, the RVB mean-field pairing amplitude $\Delta_{0}$ should slightly increase toward the center of the vortex.) This is qualitatively different from the behavior found in BCS superconductors where the pairing amplitude (or local gap) collapse at the center of the vortex. This difference could be manifested in the absence of vortex-core induced midgap states. 19]

At closing it is important to point out that the treatment in this paper eventually becomes invalid as $x \rightarrow 0$, where a charge $\mathrm{SU}(2)$ symmetry emerges. [7, 8] We believe that the enlarged gauge symmetry is responsible for antiferromagnetism. Moreover, the SU(2) gauge fluctuations at length scales smaller than the holon separation can cause important modifications of the bare parameters (or even introducing new spinon interaction terms) in our theory. Finally, the cutoff length and energy scales in this work can be affected by the (dynamic) stripe correlation [23] presents in the underdoped systems.

Acknowledgement I thank G. Baskaran and Steve Kivelson for useful discussions, and Xiao-Gang Wen for helpful comments. This work was supported by NSF grant DMR 99-71503.

\section{BIBLIOGRAPHY}

[1] P.W. Anderson, Science,235, 1196 (1987).

[2] D. S. Marshall et al., Phys. Rev. Lett. 76, 4841 (1996); A. G. Loeser et al., Science 273, 3235 (1996); H. Ding et al., Nature (London) 382, 51 (1996); F. Ronning et al, Science, 282, 2067 (1998).

[3] R. B. Laughlin, Phys. Rev. Lett. 79, 1726 (1997).

[4] I. Affleck and J. B. Marston, Phys. Rev. B 37, 3774 (1988).

[5] G. Kotliar, Phys. Rev. B 37, 3664 (1988); G. Kotliar and J. Liu, Phys. Rev. B 38, 5142 (1988).

[6] G. Baskaran and P.W. Anderson, Phys. Rev. B 37, 580 (1988); L. Ioffe and A. Larkin, Phys. Rev. B 39, 8988 (1989); P.A. Lee and N. Nagaosa, Phys. Rev. B 45,966 (1992).

[7] I. Affleck, Z. Zhou, T. Hsu, and P.W. Anderson, Phys. Rev. B 38, 745 (1988).

[8] See, e.g., P.A. Lee, N. Nagaosa, T-K Ng and X-G Wen, Phys. Rev. 576003 (1998).

[9] Y. Suzumura et al, J. Phys. Soc. Jpn 57, 2768 (1988); H. Fukuyama, Prog. Theo. Phys. Suppl. 108, 287 (1992).

[10] For a review, see, e.g. P.A. Lee, cond-mat/9812226 (1998).

[11] L. Balents, M.P.A. Fisher and C. Nayak, Int. J. Mod. Phys. B 12, 1033 (1998).

[12] By performing the gauge transformation leading from "nodon" to electron in Ref. 11], it is impossible to trade the coupling between fermion and the $\phi_{p}$ in Eq. (12) with an order parameter phase.

[13] P.A. Lee and X-G Wen, Phys. Rev. Lett. 78, 4111 (1997).

[14] A.J. Millis, S.M. Girvin, L.B. Ioffe and A.I. Larkin, J. Phys. Chem. Solids, 59, 1742 (1998).

[15] J. Corson et al, Nature, 398, 221 (1999).

[16] M.U. Ubbens and P.A. Lee, Phys. Rev. B 49, 6853 (1994).

[17] The stability of the spin gap phase is also revisited in Y.B. Kim and Z. Wang, cond-mat/9901003.

[18] N. Nagaosa, Phys. Rev. B 45, 966 (1992).

[19] Ch. Renner, B. Revaz, K. Kadowaki, I. Maggio-Aprile, and . Fischer, Phys. Rev. Lett. 80, 3606 (1998).

[20] S. Doniach and M. Inui, Phys. Rev. B 41, 6668 (1990).

[21] V. J. Emery and S. A. Kivelson, Nature (London) 374, 434 (1995).

[22] Y.J. Uemura et al, Phys. Rev. Lett. 62, 2317 (1989); C.A.R. Sa de Melo, M. Randeria, and J.R. Engelbrecht, Phys. Rev. Lett. 71, 3202 (1993).

[23] V.J. Emery, S.A. Kivelson and J. Tranquada, condmat/9907228 